\title{
FROM TELLING TO SHARING TO SILENCE: A LONGITUDINAL ETHNOGRAPHY ON THE DYNAMICS OF HEALTHCARE PROFESSIONAL-PATIENT COMMUNICATION ABOUT ORAL CHEMOTHERAPEUTIC TREATMENT FOR COLORECTAL CANCER.
}

Dr Gary Mitchell, Queen's University Belfast, Northern Ireland $\mid$ Professor Sam Porter, Bournemouth University, England | Professor Elizabeth Manias, Deakin University, Australia.

Background: Healthcare professionals are encouraged to promote concordance, a shared agreement about prescription and administration of medications, in their communication with patients. However, there is a paucity of research regarding the impact of communication about selfadministered oral chemotherapy.

Aim: To examine the changing dynamics of communication through the patient journey from diagnosis of colorectal cancer to post treatment of chemotherapy.

Methods: Over 60 hours of observational data were digitally recorded from interactions between 15 healthcare professionals, 8 patients with colorectal cancer prescribed Capecitabine and 11 family members over a six-month period in outpatient departments within one hospital in the United Kingdom. Sixteen semi-structured interviews were conducted with patients during and after their treatment. Three focus-groups were carried out with healthcare professionals. These data were analysed using thematic analysis.

\section{Results:}

Theme One: Autocratic Communication Processes

Communication was almost entirely led by healthcare professionals who concentrated on medical aspects of treatment such as how to administer capecitabine safely, how to recognise side-effects and when to contact a 24-hour chemotherapy help-line.

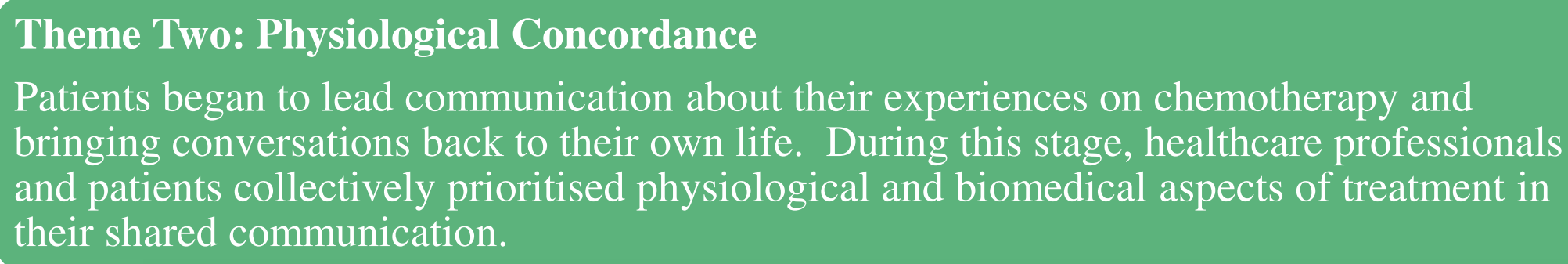

Theme Three: Holistic Concordance

Patients and families communicated about psychosocial issues of chemotherapy

management. This dialogue took the form of expressing sadness, uncertainty and anger

about living with cancer, sexual dysfunction, hair-loss and adjustments required for going

back to work in the future

Theme Four: Silence

Patients felt isolated and unsupported at the end of their treatment. This was because once

treatment was complete, patients no longer seen their oncology team on a regular basis.

Therefore, there was not any opportunity to communicate post-treatment anxieties to an

oncology team

Discussion:

1. In the early stage following cancer diagnosis, the central goal of consultations with healthcare professionals was to educate patients and their family about the practicalities of oral chemotherapy. Patients and their families spoke highly of the competence of their oncology team at this stage of their journey. For all participants, the biological needs of patients tended to supersede psychological, social and spiritual needs.

2. As patients became more familiar with their treatment regimen, they began to take greater control of their consultations. Initially, this control manifested as an increased focus on the physiological side-effects that the patient was experiencing and how these could be medically managed. Communication from healthcare professionals was subsequently directed on the physical impact of chemotherapy and side-effects, such as diarrhoea, nausea, vomiting, and erythema, and the ways in which these side-effects were managed by pharmacological interventions.

3. Towards the end of their journey, patients were facilitated to discuss their experiences of taking chemotherapy in the context of their own life through topics like; managing hair loss, going on family holidays, attending social events, returning to work and managing fatigue. Healthcare professionals supported patients to talk about their unique psychological and social experiences in the context of their treatment.

4. At the end of treatment, patients felt they faced an uncertain future because regular communication with their healthcare professional team had come to an end. 